\title{
Análise fatorial confirmatória da Escala de Ageismo no Contexto Organizacional
}

\author{
Andreia da Rocha Siqueira-Brito, Lucia Helena Freitas Pinho França ${ }^{1}$, Felipe Valentini \\ Universidade Salgado de Oliveira, Niterói-RJ, Brasil
}

\section{RESUMO}

Este estudo analisou a estrutura da Escala de Ageismo no Contexto Organizacional (EACO). Participaram 383 trabalhadores do Estado do Rio de Janeiro, de 18 a 75 anos $(M=40 ; D P=12,79)$, de ambos os sexos e diferentes níveis de escolaridade, sendo utilizada a análise fatorial confirmatória (AFC). Os resultados das AFCs foram satisfatórios e o modelo de dois fatores independentes apresentou indicadores de ajuste aceitáveis $\left(\chi^{2}=208,08\right.$; Tucker-Lewis Index $-\mathrm{TLI}=0,93$; Comparative Fit Index - CFI=0,94; Root-Mean-Square Error of Approximation - RMSEA=0,06), sustentando quase totalmente a estrutura da análise exploratória da escala original, sendo retirado apenas um item. Assim, a EACO constou de 13 itens, subdivididos em: Dimensão 1 - atitudes positivas; e Dimensão 2 atitudes negativas, na qual os trabalhadores jovens apresentaram atitudes mais negativas quanto ao envelhecimento nas organizações, confirmando estudo prévio. Recomenda-se a reaplicação dessa escala em outros contextos para investigar causas e consequências do ageismo organizacional.

Palavras-chave: envelhecimento; escalas; preconceito, grupos etários; organizações.

\section{ABSTRACT - Confirmatory factor analysis of the Ageism scale in the Organizational Context}

This study analyzed the structure of the Ageism Scale in the Organizational Context (EACO) with 383 workers from the State of Rio de Janeiro, Brazil, aged between 18 and 75 years $(M=40 ; S D=12.79)$, of both sexes and different educational levels, using confirmatory factor analysis (CFA). Results of the CFA were satisfactory, revealing that the two independent factor model presented acceptable adjustment indicators $\left(\chi^{2}=208.08\right.$; Tucker-Lewis Index - TLI=0.93; Comparative Fit Index - CFI=0.94; Root-MeanSquare Error of Approximation - RMSEA=0.06) giving almost total support to the original scale structure with only one item withdrawn. Thus, the EACO with workers in Rio de Janeiro consisted of 13 items, subdivided in: Dimension 1 - positive attitudes; and Dimension 2 - negative attitudes, confirming previous study in which younger workers showed more negative attitudes towards ageing in the organizations. Reapplication of EACO in other organizational contexts to investigate causes and consequences of ageism is recommended.

Keywords: ageing; scaling (testing); prejudice; developmental age groups; organizations.

\section{RESUMEN - Análisis factorial confirmatorio de la Escala del Ageísmo en el contexto organizacional}

Este estudio analizó la estructura de la Escala del Ageísmo en el contexto organizacional. Un total de 383 trabajadores del Estado del Rio de Janeiro, entre 18 y 75 años $(M=40 ; D E=12,79)$, de ambos géneros y diferentes niveles educativos participaron del estudio. Se utilizó el análisis factorial confirmatorio. Los resultados del análisis fueron satisfactorios, y el modelo de dos factores independientes presentó indicadores de ajuste aceptables $\left(\chi^{2}=208,08\right.$; índice de Tucker-Lewis=0,93; Comparative Fit Index=0,94; Root-Mean-Square Error of Approximation =0,06), dando apoyo casi total a la estructura del análisis exploratorio de la escala original, con sólo un ítem retirado. Así, el Escala del Ageísmo en el contexto organizacional constaba de 13 ítems, subdivididos en: Dimensión 1 - actitudes positivas; y Dimensión 2 - actitudes negativas, en la cual los trabajadores más jóvenes mostraron actitudes más negativas hacia el envejecimiento en las organizaciones, lo que confirma un estudio previo. Se recomienda una nueva aplicación de la Escala del Ageísmo en otros contextos organizacionales para investigar las causas y las consecuencias de la discriminación organizacional por edad. Palabras clave: envejecimiento; escalas; prejuicio; grupos por edad; organizaciones.

O fenômeno da mudança demográfica, caracterizada pelo aumento do número de pessoas mais velhas e pela redução das taxas de natalidade, é observado no mundo inteiro. Esse fenômeno ocasionará, em consequência, uma redução no número de trabalhadores de 14 a 64 anos (ou a população economicamente ativa).
Assim, cada vez mais os trabalhadores mais velhos precisarão estender o seu período de trabalho por mais tempo (França, 2012; Lima, Carvalho-Neto, \& Tanure, 2012; Goldani, 2010; Van Dalen, Henkens, \& Schippers, 2010).

Para que haja a continuidade dos trabalhadores mais velhos no mercado de trabalho e seja criada uma cultura 
de diversidade intergeracional, é preciso que as organizações, por meio dos seus recursos humanos, integrem a faixa etária dos mais jovens com os mais velhos, de forma que os benefícios dessa diversidade, a harmonia da equipe e a solidariedade intergeracional sejam propiciados (Celis, Pinedo, Vélez, Rodriguez, \& Saavedra, 2012; Loth \& Silveira, 2014).

No que diz respeito à academia, são igualmente necessárias a promoção de pesquisas e a discussão sobre o desafio do envelhecimento nas organizações, visando tanto o bem-estar dos trabalhadores que se aposentam quanto a possibilidade de trabalho para aqueles que desejam continuar trabalhando, mesmo após a aposentadoria (França, 2012). Entretanto, um dos maiores impedimentos tanto para a continuidade como para (re)absorção desses trabalhadores mais velhos pelo mercado tem sido o preconceito contra a idade, ou ageismo, como assinalado por Loth e Silveira (2014).

A Associação de Psicologia Americana (APA) aponta que a idade é um importante elemento da diversidade, sendo necessário que as instituições de psicologia rejeitem a discriminação baseada nesse componente e trabalhem para que o ageismo seja banido na sociedade (APA, 2002). Contudo, a quem nos referimos quando utilizamos o termo "velho"?

Existem critérios e variáveis que definem o que é ser velho, idoso ou "envelhescente". O conceito de velhice está relacionado a variáveis como gênero, raça, etnia, classe social, localização geográfica e o contexto histórico e social em que se estuda o processo de envelhecimento (Neri, 2007). No contexto organizacional, é preponderante analisar a influência que o tipo de trabalho exerce sobre o processo de envelhecimento para alguns trabalhadores, bem como os diferentes limites e as definições das organizações internacionais que se utilizam da idade para definir o velho.

Por exemplo, a Organização das Nações Unidas (ONU, 1982) admite que idoso é aquele que tem 60 anos ou mais. A Organização Mundial da Saúde (OMS, 2002) se baseia no local onde o indivíduo vive para definir o termo: em países em desenvolvimento o idoso é aquele que tem 60 anos ou mais; em países desenvolvidos, o idoso tem 65 anos ou mais. No Brasil, o Estatuto do Idoso define como idosa a pessoa com 60 ou mais (Brasil, 2003).

Sob o ponto de vista do trabalho, nem todo aposentado é velho e nem todo velho é aposentado, existindo pessoas que se aposentam e continuam trabalhando e outras que nunca receberão a justa aposentadoria por razões diversas. Algumas pessoas são consideradas velhas para um trabalho, como o caso de esportistas, visto que necessitam da força física, enquanto para outras, como os juízes ou professores, a idade incorpora a figura de sábio. No Brasil, ainda há limites de idade para a aposentadoria, especialmente no poder público, embora o Estatuto do Idoso condene qualquer discriminação de idade.
Tais contradições são observadas frequentemente nas atitudes diante do envelhecimento dos trabalhadores.

O estudo das atitudes é um dos temas mais relevantes da Psicologia Social, e sua importância hoje é ainda maior, visto que a diversidade de gênero, raça, nacionalidade, status social e idade é cada vez mais evidente. As atitudes podem ser definidas como uma organização de crenças e cognições, dotada de carga afetiva pró ou contra um objeto social definido, coerente com as cognições e os afetos relativos a esse objeto (Rodrigues, Assmar, \& Jablonski, 2000). No que diz respeito às atitudes contra a idade, ainda são raras as pesquisas sobre as atitudes negativas diante do envelhecimento (ageismo). Mas o que significa o ageismo e o que tem sido feito para evitá-lo, já que todos nós estamos envelhecendo rapidamente?

Butler (1980) afirmou que ageismo é o processo de estereotipar sistematicamente e discriminar pessoas pelo simples fato de elas serem velhas, podendo afetar desde decisões de contratação e demissão até cuidados médicos e política social. Abreu e Peixoto (2009) ressaltaram que o ageismo exclui o trabalhador mais velho do mercado. Koch-Filho et al. (2012) afirmaram que a falta de conhecimento acerca do envelhecimento pode comprometer o desenvolvimento saudável do idoso, à medida que são geradas crenças que reforçam a desvalorização e enfraquecem as oportunidades sociais e de saúde.

Goldani (2010) afirmou que a diferença entre o ageismo e a discriminação por idade é que o primeiro refere-se à avaliação do segundo. Entender o processo de envelhecimento humano pode influenciar positivamente na redução dos preconceitos, além de garantir os direitos e o bem-estar dos mais velhos (Goldani, 2010; Neri \& Jorge, 2006). Para Palmore (1999, p. 4), o ageismo é definido como "qualquer preconceito ou discriminação contra ou a favor de qualquer grupo etário". Desde 1994, o dicionário Webster (Merriam-Webster, 1994) relacionava o termo ageismo como "preconceito contra os idosos".

O ageismo, o racismo e o sexismo formam os três maiores "ismos", merecendo, por esse motivo, a mesma atenção (França, 2012; Nelson, 2005; Palmore, 1999). Torres, França, Oliveira e Presotti (2016) enfatizam que os "ismos" organizacionais se caracterizam basicamente pelas diferenças entre os grupos nas organizações. Pesquisas sobre o ageismo ainda são raras e tornam-se cada vez mais necessárias, visto que o preconceito contra os mais velhos é uma realidade que está em diversos contextos, dentre eles o ambiente de trabalho (Bodner, 2009; Wilkinson \& Ferraro, 2002).

Os estereótipos ageístas estão presentes nas organizações e influenciam o empregador na hora da contratação de empregados mais velhos (Berger, 2009; Gray \& McGregor, 2003; Nelson, 2005; Van Dalen et al., 2010). Tais posturas estereotipadas tentam modular a forma como os trabalhadores mais velhos agem e se comunicam dentro das organizações. De acordo com McCann e Giles (2004), os estereótipos negativos incluem 
irritabilidade, excentricidade, fraqueza e deficiência cognitiva. Nussbaum, Pitts, Huber, Krieger e Ohs (2005) citam outros estereótipos, como inflexibilidade, improdutividade e fragilidade, atribuídos aos trabalhadores mais velhos.

Estudos realizados por McMullin e Marshall (2001) apontaram que os trabalhadores mais velhos se percebem menos poderosos e produtivos, mas consideram-se mais experientes, responsáveis e cometem menos erros do que os trabalhadores mais jovens. Gray e McGregor (2003) constataram a eficácia dos estereótipos negativos em relação a empregados e empregadores. Esses dois grupos concordaram que os trabalhadores mais velhos são mais difíceis de treinar, têm menos disposição para aprender e mais medo de novas tecnologias. De acordo com Dennis e Thomas (2007), os gestores valorizam a experiência, o conhecimento, as atitudes, o compromisso, a lealdade, a pontualidade e a habilidade para resolver problemas dos trabalhadores mais velhos. Contudo, avaliam esses trabalhadores negativamente em relação à flexibilidade, às novas tecnologias, à resistência às mudanças, às limitações físicas, entre outros aspectos.

Van Dalen et al. (2010) realizaram um estudo sobre as características relacionadas à produtividade de trabalhadores mais velhos e mais jovens. Os resultados revelaram que as características macias (compromisso com a organização, confiabilidade e habilidades sociais) foram associadas aos trabalhadores mais velhos, e as características duras (flexibilidade, capacidade física e mental, desejo de aprender e habilidades tecnológicas) aos trabalhadores mais jovens.

França et al. (2014) reaplicaram a pesquisa de Van Dalen et al. (2010) com gestores brasileiros e seus resultados foram similares aos encontrados pelos pesquisadores holandeses, quanto à avaliação das características macias e duras de trabalhadores mais velhos e mais jovens, respectivamente. Contudo, a grande diferença entre os dois estudos é que os gestores europeus já tomaram as medidas para acomodar os trabalhadores mais velhos no mercado de trabalho, intensificando o emprego de meio período, a aposentadoria gradual e a redução da carga de trabalho, ao passo que para os gestores brasileiros essas medidas não seriam sequer consideradas no futuro.

O estudo de Celis et al. (2012) sobre as atitudes diante do envelhecimento em profissionais da saúde apontou que as pessoas mais velhas avaliam os trabalhadores idosos mais positivamente. Os resultados dos pesquisadores peruanos foram corroborados pelo estudo de França et al. (2015), que verificaram atitudes mais positivas em relação aos mais velhos por parte dos participantes com mais idade. Contrariando esses resultados, Ferreira e Félix Neto (2012), em pesquisa com portugueses de diversas faixas etárias, constataram que o grupo composto por idosos manifestou maiores atitudes preconceituosas em relação ao envelhecimento.
No que se refere ao sofrimento provocado pelo ageismo nas organizações, o pesquisador espanhol Alcover (2012) argumenta que os trabalhadores mais velhos são mais afetados pelas incertezas e inseguranças vivenciadas no ambiente de trabalho do que os trabalhadores mais jovens.

Apesar de ainda raros, alguns estudos brasileiros sobre ageismo devem ser destacados. O estudo qualitativo de Loth e Silveira (2014) relacionou os estereótipos percebidos por "envelhescentes" em relação ao preconceito contra a idade no trabalho. Os autores identificaram três categorias de percepções estereotipadas: de si mesmos, dos outros e dos jovens. França et al. (2015) e Moura e Viana (2011) defendem que os estereótipos, sejam eles negativos ou positivos, são percebidos pelos trabalhadores mais jovens e mais velhos e podem influenciar as relações de trabalho, como, por exemplo, as oportunidades de emprego negadas em função da idade. Loth e Silveira (2014) apontam para a relevância de políticas intergeracionais que promovam a aproximação entre as pessoas que se encontram em distintos estágios da vida.

Marques, Batista e Da Silva (2012) ressaltam que ao associarmos trabalhadores mais velhos aos estereótipos negativos, como "ultrapassados" e "adoecidos", dificilmente admitiremos a possibilidade de mantê-los ativos no mercado de trabalho, especialmente em cargos que exigem maior responsabilidade. Por outro lado, Lima et al. (2012) afirmam que os trabalhadores mais jovens estão mais insatisfeitos em relação à sobrecarga de trabalho, ao nível de estresse e de cobranças de resultados e à saúde de modo geral. Esses autores afirmam que os mais jovens se consideram mais pressionados a equilibrar a vida profissional e pessoal porque seus filhos ainda são pequenos. Apesar disso, os mais jovens aceitam trabalhar mais ganhando menos do que os mais experientes, o que pode provocar uma dificuldade na recolocação dos trabalhadores mais velhos no mercado de trabalho. Lima et al. (2012) revelaram ainda que os trabalhadores mais velhos se consideram mais leais do que os mais jovens, enquanto esses dizem vivenciar preconceitos associados à falta de competência e consideram os mais velhos mais resistentes às mudanças e mais presos às práticas de gestão ultrapassadas.

A escassez de instrumentos para medir o ageismo, especialmente nas organizações, deu origem à pesquisa realizada por França et al. (2015), que construíram e validaram a Escala de Ageismo no Contexto Organizacional (EACO). Esses pesquisadores definiram o ageismo no contexto organizacional como um conjunto de atitudes negativas ou positivas diante do envelhecimento, valorizando ou desvalorizando a força de trabalho mais velha; favorecendo ou não a sua inclusão no mercado de trabalho. Esses autores argumentam que quanto mais atitudes negativas a pessoa demonstrar diante do envelhecimento, mais ageísta ela será. De forma oposta, quanto menos atitudes negativas (ou mais positivas) ela apresentar, menos ageísta será. 
O estudo de França et al. (2015) contou com 600 trabalhadores de diversas idades em organizações brasileiras. Assim, o objetivo do presente trabalho foi dar continuidade à pesquisa de França et al. (2015) buscando novas evidências acerca da estrutura da EACO, por meio da Análise Fatorial Confirmatória (AFC), com trabalhadores do Estado do Rio de Janeiro. Buscou-se, ainda, identificar se a idade e os níveis de escolaridade influenciariam as atitudes negativas ou positivas diante do envelhecimento da força de trabalho no contexto organizacional. Para atingir essas metas, foram formuladas duas hipóteses:

- H1: trabalhadores mais jovens têm mais atitudes negativas e menos atitudes positivas em relação aos trabalhadores mais velhos; e

- H2: trabalhadores com menor nível de escolaridade têm mais atitudes negativas e menos atitudes positivas em relação aos trabalhadores mais velhos.

\section{Método}

\section{Participantes}

A amostra foi composta por 383 trabalhadores, com idades variando de 18 a 75 anos $(M=40 ; D P=12,79)$, sendo mais da metade composta por participantes do sexo feminino (61\%) e casados (55\%). No que diz respeito à escolaridade, $6 \%$ possuíam ensino fundamental, $32 \%$, ensino médio, $28 \%$, ensino superior, e $34 \%$, possuíam pós-graduação. Em relação ao tipo de organização, $70 \%$ trabalhavam em empresas privadas, quase a metade (47\%), em organizações com mais de 500 empregados. Os setores da economia mais representados neste estudo foram: educação (27\%), comércio (17\%) e saúde/serviço social $(15 \%)$. A maior parte dos trabalhadores $(70 \%)$ não exercia cargo de chefia.

\section{Instrumentos}

Nesta pesquisa foi utilizada a EACO (França et al., 2015). O participante deveria avaliar os trabalhadores mais velhos e mais jovens em relação a: adoecimento, rapidez ou lentidão no desenvolvimento das tarefas, produtividade, compreensão das rotinas, capacidade de concentração e memória, comprometimento, habilidade na resolução de problemas, persistência, conhecimento, capacidade para lidar com pressões do trabalho e assiduidade, comparando uma faixa etária a outra. Foi esclarecido aos participantes que os trabalhadores mais velhos representavam pessoas com 60 anos ou mais. Já os trabalhadores mais jovens representavam as pessoas entre 18 e 35 anos. Ao final no questionário, foram solicitadas informações sociodemográficas, tais como: sexo, idade, escolaridade, estado civil, tipo e tamanho da organização, setor da economia, além de uma questão sobre o exercício ou não da função de chefia.

No processo de construção da EACO (França et al., 2015), a escala foi composta por 28 itens, resultado da realização de pré-testes para medir a compreensão do instrumento, análise de juízes em função de uma categorização teórica e se os itens estavam claros e faziam sentido em cada categoria. Contudo, após a análise fatorial exploratória foram eliminados 14 itens, que apresentaram problemas psicométricos, emergindo uma escala breve de 14 itens, dispostos em duas dimensões:

1. atitudes negativas diante do envelhecimento $(\alpha=0,83)$, com cargas fatoriais variando entre 0,53 e 0,64 , abrangendo 9 itens, definidos principalmente por aspectos cognitivos e de saúde. Um exemplo de item é: "Trabalhadores mais velhos costumam adoecer com mais facilidade";

2. atitudes positivas $(\alpha=0,77)$ e cargas fatoriais que variam de 0,57 a 0,71 , abrangendo 5 itens, definidos principalmente por aspectos afetivos. Um exemplo de item é: "Trabalhadores mais velhos são mais comprometidos com o trabalho do que os mais jovens". As respostas são assinaladas em uma escala do tipo Likert de (1) "discordo totalmente" a (5) "concordo totalmente".

\section{Procedimentos}

Este estudo foi submetido ao Comitê de Ética em Pesquisa (CEP) da Universidade Salgado de Oliveira (UNIVERSO), por meio da Plataforma Brasil, e aprovado sob o CAAE no 26403813.4.0000.5289. Os participantes tiveram acesso ao Termo de Consentimento Livre e Esclarecido (TCLE) contendo todas as informações necessárias sobre o procedimento realizado e garantindo o sigilo e o anonimato das informações.

A coleta de dados ocorreu em duas etapas, on-line e com material impresso, com a participação de 391 trabalhadores. A coleta de dados on-line ocorreu por meio do preenchimento do questionário hospedado pelo site da Qualtrics, realizada em conjunto com outra pesquisa em âmbito nacional: "Ageismo nas organizações brasileiras: a percepção dos trabalhadores". Foram, então, enviados 1.240 convites eletrônicos para trabalhadores do Estado do Rio de Janeiro para que preenchessem o questionário de pesquisa on-line. Esse procedimento reuniu 202 questionários eletrônicos de participantes do Rio de Janeiro. Considerando que a coleta on-line obteve grande parte de trabalhadores com nível superior, realizamos uma coleta de dados complementar por meio de material impresso com trabalhadores do comércio e da indústria localizados próximos à universidade. Assim, 189 trabalhadores responderam o questionário com a ajuda de entrevistadores, dado o nível mais baixo de instrução desses participantes.

\section{Análise dos Dados}

Foram efetuadas análises exploratórias dos dados, para avaliar a ocorrência de erros de digitação e casos ausentes (missing). Dos 391 questionários respondidos, 61 (15,6\%) apresentaram missing. Foram excluídos 8 participantes: 5 casos que não responderam a idade e outros 3 que apresentaram mais de $5 \%$ de dados ausentes. 
Nos demais casos, 53 (13,6\%), os dados ausentes foram substituídos pela média da variável. Após a limpeza do banco de dados, a amostra total foi de 383 participantes.

O modelo de estrutura de dois fatores apresentado por França et al. (2015) foi testado nesta pesquisa por meio da Modelagem por Equações Estruturais (MEE). Considerando a natureza ordinal dos itens do instrumento, os parâmetros foram estimados por meio do método Weighted Least Squares Mean and Variance Adjusted, a partir de correlações policóricas. $O$ ajuste do modelo foi avaliado conforme os seguintes indicadores: $\chi^{2}$ (testa a probabilidade do modelo teórico se ajustar aos dados, no qual quanto maior o valor do $\chi^{2}$, pior o ajustamento); Root-Mean-Square Error of Approximation (RMSEA - deve se situar abaixo de 0,05, aceitando-se valores até 0,08); Tucker-Lewis Index (TLI - considera adequados os valores acima de 0,95); Comparative Fit Index (CFI - considera adequado que os valores estejam acima de 0,95) (Byrne, 2012).

A consistência interna da escala foi estimada por meio do alfa de Cronbach. Foram realizadas as correlações de Pearson entre as variáveis sociodemográficas e as dimensões da EACO e, a seguir, as análises de regressão linear múltipla. Tais análises foram feitas com o auxílio dos softwares SPSS (versão 19) e Mplus (versão 7.11).

\section{Resultados}

Foram realizadas análises descritivas para determinar a frequência, a moda e a mediana dos itens em relação à amostra estudada. A maior frequência foi observada na resposta 1 do item 3 (214); na maioria dos itens, a moda ficou concentrada na resposta 3 e a mediana variou entre as respostas 2 e 3 . É interessante notar que cinco itens obtiveram mais da metade dos seus resultados concentrados na discordância total ou discordância (1 e 2); foram os itens 3,4 e 26, que diziam respeito às faltas, ao esquecimento e aos acidentes de trabalho atribuídos aos idosos, e os itens 4 e 27, que atribuíam aos jovens maior capacidade de concentração e maior compreensão das rotinas. Esses resultados parecem quebrar os rótulos negativos atribuídos aos idosos (Tabela 1).

Os resultados evidenciaram que o modelo de dois fatores apresentava indicadores de ajuste aceitáveis $\left[\chi^{2}=208,08 ; \quad \mathrm{TLI}=0,93 ; \mathrm{CFI}=0,94 ; \quad\right.$ RMSEA $(\mathrm{IC} 90 \%)=0,06(0,06-0,08)]$. Contudo, os itens 26 e 27 apresentaram covariância residual; assim, optou-se pela exclusão do item 27, visto que apresentou carga fatorial mais baixa. Os resultados das cargas fatoriais e dos indicadores de ajuste para o modelo final (sem o item 27) são apresentados na Tabela 2.

No modelo final, apresentado na (Tabela 2), as variâncias das variáveis latentes foram fixadas em 1 , para que todos os coeficientes de regressão entre as variáveis latentes e os itens fossem livremente estimados. Assim, os coeficientes de regressão "não padronizados" apresentaram valores iguais aos padronizados. Conforme resultados apresentados na Tabela 2, todos os parâmetros não padronizados foram estatisticamente diferentes de 0 (i.e. o intervalo de confiança não contém o valor 0 ). Verificase, ainda, que os 13 itens apresentaram cargas fatoriais acima de 0,60 $(M=0,63$ para a Escala Total; $M=0,61$ para o Fator 1; $M=0,66$ para o Fator 2), exceto os itens 4 e 11, cujas cargas fatoriais foram iguais a 0,48 e 0,56 , respectivamente.

No que se refere ao ajuste do modelo, ainda que o $\chi^{2}$ tenha sido estatisticamente significativo $(p>0,05)$, os demais indicadores apontam para um ajuste plausível (minimamente aceitável) do modelo aos dados empíricos.

Em resumo, o modelo foi composto por duas dimensões: a primeira dimensão de atitudes negativas (D1), composta por oito itens; e a segunda dimensão de atitudes positivas (D2), composta por cinco itens, como pode ser observado na Tabela 2. A consistência interna da escala foi obtida pelo alfa de Cronbach, sendo o seu resultado de 0,78 para atitudes negativas e de 0,75 para atitudes positivas.

Foram realizadas correlações de Pearson entre os dados sociodemográficos (idade e escolaridade) e as dimensões da escala. A idade e a escolaridade se correlacionaram significativamente com as atitudes negativas e positivas da EACO. A idade apresentou uma correlação negativa em relação às atitudes negativas, ou seja, quanto maior a idade, menores são os escores de atitudes negativas. A idade apresentou ainda uma correlação positiva referente às atitudes positivas, indicando que quanto maior a idade, mais atitudes positivas são observadas. Entretanto, é necessário destacar que os tamanhos de efeito dessas correlações foram baixos $(r=-0,16$ para atitudes negativas e $r=0,18$ para atitudes positivas). Tais efeitos indicam que a idade e o ageismo compartilham menos de $4 \%$ de variância. Portanto, futuros estudos são necessários para corroborar essa relação com outros grupos de trabalhadores.

No que tange ao nível de escolaridade, os resultados apontaram uma correlação negativa diante das duas dimensões da escala, ou seja, quanto menor o nível de escolaridade, mais atitudes negativas e positivas em relação ao envelhecimento do trabalhador na organização. Esses resultados confirmam parcialmente H2. Conforme apontado, é necessária uma nova aplicação dessa escala em outra amostra, de forma a aprofundar essa ambivalência.

A fim de observar o poder preditivo das variáveis idade e escolaridade em função das dimensões do ageismo organizacional (atitudes negativas - D1; atitudes positivas - D2), foram realizadas duas regressões lineares múltiplas utilizando a amostra total da pesquisa $(n=383)$.

Em relação às atitudes negativas, o conjunto de variáveis independentes (VIs), formado pela idade/escolaridade, explicou cerca de $5 \%$ da D1 $\left(R^{2}=0,054 ; F=10,79\right.$; $p<0,001)$. Os resultados foram significativos para a idade $\mathrm{e}$ a escolaridade quanto às atitudes negativas. Ou seja, quanto menores a idade $(\beta=-0,13 ; t=-2,58 ; p<0,05)$ e a escolaridade $(\beta=-0,17 ; t=-3,24 ; p<0,01)$ daquele que é submetido 
à escala, mais atitudes negativas diante do envelhecimento dos trabalhadores nas organizações são observadas. No que tange às atitudes positivas, as VIs explicaram aproximadamente $6 \%$ da D2 $\left(R^{2}=0,059 ; F=11,96 ; p<0,001\right)$. A idade e a escolaridade também apresentaram resultados significativos quanto às atitudes positivas, indicando que quanto maior a idade $(\beta=0,21 ; t=4,16 ; p<0,001)$ e menor a escolaridade $(\beta=-0,17 ; t=-3,38 ; p<0,01)$ de quem se submete à escala, mais atitudes positivas ele(a) tem diante do envelhecimento na organização. Os resultados das regressões e correlações estão dispostos na Tabela 3.

\section{Discussão}

O ageismo nas organizações se constitui em um importante fator de exclusão dos trabalhadores mais velhos (Abreu \& Peixoto, 2009; Berger, 2009; Gray \&
McGregor, 2003; Van Dalen et al., 2010). Estudos brasileiros sobre esse tema ainda são escassos; essa falta de conhecimento tende a reduzir as oportunidades de inclusão e a permanência dos trabalhadores mais velhos no mercado de trabalho, como apontado por França et al. (2015), Koch-Filho et al. (2012), Loth e Silveira (2014), Marques et al. (2012) e Moura e Viana (2011).

Uma das formas de inclusão dos trabalhadores mais velhos nas organizações é identificar preconceitos existentes contra a idade e definir estratégias facilitadoras de integração dos grupos intergeracionais. A gestão de recursos humanos deve ser estimulada a trabalhar junto com a comunidade acadêmica, focando na diversidade da sua organização, tendo em suas mãos estudos, pesquisas, medidas e intervenções que possam identificar as raízes das discriminações, os mecanismos, as possibilidades e as estratégias para o fomento da inclusão.

Tabela 1

Análise descritiva dos itens da Escala de Ageismo no Contexto Organizacional $(n=383)$

\begin{tabular}{|c|c|c|c|c|c|c|c|}
\hline \multirow[t]{2}{*}{ Itens } & \multicolumn{5}{|c|}{$\begin{array}{c}\text { Frequência por } \\
\text { categoria de resposta (Likert) }\end{array}$} & \multirow[t]{2}{*}{ Moda } & \multirow[t]{2}{*}{ Mediana } \\
\hline & 1 & 2 & 3 & 4 & 5 & & \\
\hline $\begin{array}{l}\text { 1. Trabalhadores mais velhos levam mais tempo } \\
\text { para realizar tarefas no ambiente de trabalho }\end{array}$ & 104 & 92 & 123 & 39 & 25 & 3 & 2 \\
\hline $\begin{array}{l}\text { 2. Trabalhadores mais velhos costumam adoecer } \\
\text { com mais facilidade }\end{array}$ & 80 & 85 & 106 & 77 & 35 & 3 & 3 \\
\hline $\begin{array}{l}\text { 3. Trabalhadores mais velhos costumam faltar } \\
\text { mais ao trabalho }\end{array}$ & 214 & 88 & 50 & 19 & 12 & 1 & 1 \\
\hline $\begin{array}{l}\text { 4. Trabalhadores mais jovens costumam ter maior } \\
\text { capacidade de concentração }\end{array}$ & 113 & 104 & 96 & 45 & 25 & 1 & 2 \\
\hline $\begin{array}{l}\text { 7. Os trabalhadores mais velhos tendem a } \\
\text { esquecer novas tarefas }\end{array}$ & 120 & 115 & 85 & 37 & 26 & 1 & 2 \\
\hline $\begin{array}{l}\text { 10. Trabalhadores mais velhos são mais } \\
\text { persistentes do que os mais jovens }\end{array}$ & 33 & 48 & 109 & 105 & 88 & 3 & 4 \\
\hline $\begin{array}{l}\text { 11. Trabalhadores mais jovens são mais produtivos } \\
\text { do que os mais velhos }\end{array}$ & 89 & 93 & 136 & 42 & 23 & 3 & 3 \\
\hline $\begin{array}{l}\text { 15. Trabalhadores mais velhos são mais } \\
\text { comprometidos com o trabalho do que os } \\
\text { mais jovens }\end{array}$ & 47 & 51 & 113 & 86 & 86 & 3 & 3 \\
\hline $\begin{array}{l}\text { 16. De modo geral, trabalhadores mais velhos têm } \\
\text { mais conhecimento do trabalho }\end{array}$ & 34 & 56 & 97 & 107 & 89 & 4 & 4 \\
\hline $\begin{array}{l}\text { 18. Trabalhadores mais velhos são mais capazes } \\
\text { de lidar com pressões do trabalho }\end{array}$ & 41 & 49 & 130 & 101 & 62 & 3 & 3 \\
\hline $\begin{array}{l}\text { 20. O envelhecimento afeta a produtividade dos } \\
\text { trabalhadores }\end{array}$ & 95 & 104 & 107 & 54 & 23 & 3 & 2 \\
\hline $\begin{array}{l}\text { 23. Trabalhadores mais velhos têm mais } \\
\text { habilidade para resolver problemas do que os } \\
\text { mais jovens }\end{array}$ & 33 & 57 & 135 & 87 & 71 & 3 & 3 \\
\hline $\begin{array}{l}\text { 26. Trabalhadores mais velhos tendem a sofrer } \\
\text { mais acidentes de trabalho do que os mais jovens }\end{array}$ & 132 & 91 & 104 & 30 & 26 & 1 & 2 \\
\hline $\begin{array}{l}\text { 27. Trabalhadores mais jovens compreendem } \\
\text { melhor as rotinas de trabalho do que os mais velhos }\end{array}$ & 134 & 84 & 109 & 37 & 19 & 1 & 2 \\
\hline
\end{tabular}

Nota: os itens variam de (1) "discordo totalmente" a (5) "concordo totalmente". 
A fim de ampliar o conhecimento sobre o ageismo no contexto organizacional, este estudo objetivou analisar a estrutura da EACO (França et al., 2015) e verificar se a idade e o nível de escolaridade influenciam as atitudes negativas e positivas diante do envelhecimento da força de trabalho no ambiente organizacional. Foram realizadas AFCs, e os resultados foram bastante satisfatórios, uma vez que sustentaram, quase por completo, a estrutura da escala construída por França et al. (2015), com a retirada de apenas um item (27): "Trabalhadores mais jovens compreendem melhor as rotinas de trabalho do que os mais velhos".
As correlações de Pearson e as análises de regressão linear múltipla demonstraram que as variáveis idade e escolaridade são preditoras das atitudes negativas e positivas diante do envelhecimento do trabalhador na organização. No que tange à idade e ao ageismo, a percepção dos trabalhadores mais jovens sugere uma postura mais preconceituosa, visto que apresentaram atitudes mais negativas e menos positivas diante do envelhecimento dos trabalhadores, fato observado também nos estudos de França et al. (2015), Loth e Silveira (2014), Celis et al. (2012), Lima et al. (2012) e Van Dalen et al. (2010), sobretudo

Tabela 2

Parâmetros e indicadores de ajuste da análise fatorial confirmatória

\begin{tabular}{|c|c|c|c|c|}
\hline \multirow{2}{*}{ Parâmetros } & \multirow{2}{*}{$\beta$} & \multirow{2}{*}{ Erro padrão } & \multicolumn{2}{|c|}{ IC95\% } \\
\hline & & & Mínimo & Máximo \\
\hline At. Negat. $\rightarrow$ Age. 01 & 0,67 & 0,03 & 0,60 & 0,73 \\
\hline At. Negat. $\rightarrow$ Age. 02 & 0,67 & 0,04 & 0,60 & 0,74 \\
\hline At. Negat. $\rightarrow$ Age. 03 & 0,61 & 0,04 & 0,53 & 0,69 \\
\hline At. Negat. $\rightarrow$ Age. 04 & 0,48 & 0,04 & 0,40 & 0,57 \\
\hline At. Negat. $\rightarrow$ Age. 07 & 0,62 & 0,04 & 0,55 & 0,69 \\
\hline At. Negat. $\rightarrow$ Age. 11 & 0,56 & 0,04 & 0,49 & 0,64 \\
\hline At. Negat. $\rightarrow$ Age. 20 & 0,64 & 0,03 & 0,57 & 0,70 \\
\hline At. Negat. $\rightarrow$ Age. 26 & 0,60 & 0,04 & 0,53 & 0,67 \\
\hline At. Posit. $\rightarrow$ Age. 10 & 0,65 & 0,04 & 0,58 & 0,72 \\
\hline At. Posit. $\rightarrow$ Age. 15 & 0,73 & 0,03 & 0,67 & 0,80 \\
\hline At. Posit. $\rightarrow$ Age. 16 & 0,67 & 0,04 & 0,60 & 0,74 \\
\hline At. Posit. $\rightarrow$ Age. 18 & 0,63 & 0,03 & 0,57 & 0,70 \\
\hline At. Posit. $\rightarrow$ Age. 23 & 0,64 & 0,04 & 0,57 & 0,71 \\
\hline \multicolumn{5}{|l|}{ Correlação } \\
\hline At. Negat. $\leftrightarrow$ At. Posit. & 0,16 & 0,06 & 0,04 & 0,28 \\
\hline
\end{tabular}

Indicadores de ajuste: $\chi^{2}(\mathrm{gl})=165,45$ (64); CFI=0,95; TLI=0,94; RMSEA (IC90\%)=0,06 (0,05-0,08).

Nota: IC95\%=intervalo de confiança de 95\%; CFI=Comparative Fit Index; TLI=Tucker-Lewis Index; RMSEA=Root-Mean-Square Error of Approximation.

Tabela 3

Regressão para atitudes negativas e positivas e correlações entre as variáveis

\begin{tabular}{|c|c|c|c|c|}
\hline Variável dependente & Variável critério & $\beta$ & $t$ & $p$ \\
\hline \multirow{2}{*}{ Atitudes negativas } & Idade & $-0,13$ & $-2,58$ & 0,010 \\
\hline & Escolaridade & $-0,17$ & $-3,24$ & 0,001 \\
\hline \multirow{2}{*}{ Atitudes positivas } & Idade & 0,21 & 4,16 & 0,001 \\
\hline & Escolaridade & $-0,17$ & $-3,38$ & 0,001 \\
\hline Correlações & 1 & 2 & 3 & 4 \\
\hline 1 - Idade & - & & & \\
\hline 2 - Escolaridade & $0,21^{*}$ & - & & \\
\hline 3 - Atitudes negativas & $-0,16^{*}$ & $-0,19^{*}$ & - & \\
\hline 4 -Atitudes positivas & $0,18^{*}$ & $-0,13^{*}$ & $0,14^{*}$ & - \\
\hline
\end{tabular}

Nota: $n=383 ; R^{2}=0,054 ; F=10,79 ; p<0,001$ (atitudes negativas); $R^{2}=0,059 ; F=11,96 ; p<0,001$ (atitudes positivas); ${ }^{*} p<0,05$. 
porque os trabalhadores mais velhos são os que mais vivenciam experiências de preconceito no ambiente organizacional (Alcover, 2012). Em contrapartida, Ferreira e Félix Neto (2012) observaram maior preconceito dos idosos em relação ao seu próprio grupo.

Quanto à escolaridade e ao ageismo, os resultados foram similares aos encontrados por França et al. (2015), que observaram uma ambivalência dos trabalhadores com nível de escolaridade mais baixo, já que expressaram tanto atitudes mais negativas quanto mais positivas diante do envelhecimento. Essa ambivalência pode ter sido provocada pela ignorância em relação às possibilidades e às competências dos mais velhos (Koch-Filho et al., 2012). Em uma direção contrária, Celis et al. (2012) apontaram uma postura menos preconceituosa por parte de profissionais peruanos mais graduados.

A principal limitação deste estudo está relacionada à amostra. A coleta foi on-line, sendo a maioria dos trabalhadores com nível de instrução superior, já que o banco de dados foi formado por pessoas do círculo acadêmico que, por sua vez, repassaram o questionário aos seus colegas e alunos, acessando o link da pesquisa utilizando a técnica bola de neve. Assim, o fato de a amostra ser de conveniência gerou algumas restrições quanto a sua representatividade, embora para ampliar a representatividade da pesquisa tenha sido realizada uma segunda coleta, em questionário de papel, com trabalhadores do comércio local, próximo à universidade, grande parte deles de nível fundamental e médio.
O estudo efetuou análise confirmatória da EACO com resultados satisfatórios para um constructo recente, com número de participantes adequado, sendo uma pesquisa pioneira sobre ageismo organizacional no Rio de Janeiro. Entretanto, é fundamental que novas pesquisas sejam realizadas no sentido de ampliar o conhecimento sobre o constructo do ageismo no contexto organizacional utilizando a EACO e/ou associando-a a outras escalas que investiguem as causas e consequências do ageismo no mundo do trabalho.

O que seria um preconceito ou uma observação não tendenciosa? Acreditamos ser necessário discutir a temática do ageismo com maior frequência na academia e nas organizações. Sem dúvida, é difícil mensurar preconceitos, uma vez que as pessoas, em geral, tentam escamotear respostas que pareçam politicamente incorretas. Nesse sentido, este estudo poderá contribuir para a reflexão entre os gestores sobre os itens e as dimensões da EACO e a possível replicação dessa escala em suas organizações. Essa reflexão poderá levar ao aprimoramento desse instrumento, à criação de outros e à adoção de algumas medidas que precisam ser tomadas a fim de promover um ambiente de trabalho mais amigável. Sob o ponto de vista acadêmico, seria relevante comparar os resultados obtidos com esta pesquisa com amostras formadas por gestores de diferentes organizações, de forma a verificar as diferenças entre os dois níveis hierárquicos e se o nível de ageismo dos gestores fortaleceria ou impediria o seu papel na proposição e na adoção de políticas mais amigáveis e de inclusão para os trabalhadores mais velhos na organização.

\section{Referências}

Abreu, A., \& Peixoto, J. (2009). Demografia, mercado de trabalho e imigração de substituição: tendências, políticas e prospectiva no caso português. Análise Social, XLIV(193), 719-746. Recuperado de http://www.scielo.mec.pt/pdf/aso/n193/n193a04.pdf

Alcover, C.-M. (2012) ¿Ageism en las organizaciones? El papel mediador del apoyo organizacional percibido en las relaciones entre la edad y la ruptura del contrato psicológico. Revista Psicologia Organizações e Trabalho, 12(3), 299-313. Recuperado de http://pepsic.bvsalud.org/ scielo.php?script $=$ sci_arttext\&pid $=$ S1984-66572012000300005\&lng $=$ pt\&tlng $=\mathrm{es}$

American Psychological Association. (2002). Ethical principles of psychologists and code of conduct. American Psychologist, 57, 10601073. doi: 10.1037/0003-066x.57.12.1060

Brasil. (2003). Estatuto do Idoso. Lei no 10.741, de 1 de outubro de 2003. Brasília, DF: Presidência da República. Recuperado de http://www. planalto.gov.br/ccivil_03/LEIS/2003/L10.741.htm

Brasil. (2005). Envelhecimento ativo: uma política de saúde. Brasília: Organização Pan-Americana da Saúde.

Berger, E. D. (2009). Managing age discrimination: An examination of the techniques used when seeking employment. The Gerontologist, 49(3), 317-332. doi: 10.1093/geront/gnp031

Bodner, E. (2009). On the origins of ageism among older and younger adults. International Psychogeriatrics, 21(6), 1003-1014. doi: 10.1017/ S104161020999055X

Butler, R. N. (1980). Ageism: A foreword. Journal of Social Issues, 36(2), 8-11.

Byrne, B. M. (2012). Structural equation modeling with MPlus: Basic concepts, applications, and programming. New York: Routledge Academic.

Celis, J. D S., Pinedo, L. F. V., Vélez, C. D., Rodríguez, T. T., \& Saavedra, P. J. O. (2012). Validación de la Escala de Actitudes hacia el Adulto Mayor de Kogan y evaluación de las Actitudes hacia el adulto mayor por parte del personal de salud del primer nivel asistencial. Acta Médica Peruana, 29(3), 148-154. Recuperado de http://www.scielo.org.pe/scielo.php?script=sci_arttext\&pid=S1728$59172012000300004 \& \operatorname{lng}=\mathrm{es} \& \mathrm{nrm}=$ iso

Dennis, H., \& Thomas, K. (2007). Ageism in the workplace. Generations, 31(1), 84-89. Recuperado de http://users.manchester.edu/FacStaff/ MPLahman/Homepage/McFaddenSFMWebsiteonAgeism/ArticleDennisThomas.pdf

Ferreira, A. V., \& Félix Neto (2012). Quem são mais preconceituosos em relação à idade e os mais sós: jovens, adultos ou idosos? Influência da religiosidade. International Journal of Development and Educational Psychology, 3(1), 115-122. Recuperado de http://infad.eu/RevistaINFAD/ index.php/revista-infad-2012-no2-volumen-1-pp-115-122/ 
França, L. H. F. (2012). Envelhecimento dos trabalhadores nas organizações: estamos preparados? Em L. H. F. França \& D. V. Stepansky (Orgs.), Propostas multidisciplinares para o bem-estar na aposentadoria. (pp. 25-52). Rio de Janeiro: FAPERJ; Editora Quartet.

França, L. H. F., Nalin, C. P., Siqueira-Brito, A. R., Amorim, S. M., Rangel, T., \& Ekman, N. C. (2014). A percepção dos gestores brasileiros sobre os programas de preparação para a aposentadoria. Estudos Interdisciplinares sobre o Envelhecimento, 19(3), 879-898. Recuperado de http://seer.ufrgs.br/index.php/RevEnvelhecer/article/view/50434

França, L. H. F., Vasques-Menezes, I., Valentini, F., Nalin, C. P., Leite, S. V., Siqueira-Brito, A. R., \& Silva, L. A. (2015). Ageismo nas organizações brasileiras: a percepção dos trabalhadores (Relatório de Pesquisa/2013-2014). Universidade Salgado de Oliveira, Rio de Janeiro.

Goldani, A. M. (2010). Desafios do "preconceito etário" no Brasil. Revista Educação e Saúde, 31(111), 411-434. doi: 10.1590/S010173302010000200007

Gray, L., \& McGregor, J. (2003). Human resource development and older workers: Stereotypes in New Zealand. Asia Pacific Journal of Human Resources, 41(3), 338-353.

Koch-Filho, H. R., Koch, L. F. A., Kusma, S. M., Werneck, R. I., Bisinelli, J. C., Moysés, S. T., \& Alanis, L. R. A. (2012). Uma reflexão sobre o preconceito etário na saúde. Revista Gestão \& Saúde, 4(2), 40-48. Recuperado de http://www.herrero.com.br/revista/ edicao7artigo5.pdf.

Lima, G. S., Carvalho-Neto, A., \& Tanure, B. (2012). Executivos jovens e seniores no topo da carreira: conflitos e complementaridades. REAd Revista Eletrônica de Administração, 18(1), 63-96. doi: 10.1590/S1413-23112012000100003.

Loth, G. B., \& Silveira, N. (2014). Etarismo nas organizações: um estudo dos estereótipos em trabalhadores envelhecentes. Revista das Ciências da Administração, 39(16), 65-82. doi: 10.5007/2175-8077.2014v16n39p65

Marques, S., Batista, M., \& Da Silva, P. A. (2012). A promoção do envelhecimento ativo em Portugal. Sociologia: Revista da Faculdade de Letras da Universidade do Porto, número temático: Envelhecimento Demográfico, 53-73. Recuperado de http://ler.letras.up.pt/ uploads/ficheiros/10572.pdf

McCann, R., \& Giles, H. (2004). Ageism in the workplace: A communication perspective. Em T. D. Nelson (Ed.), Ageism: Stereotyping and prejudice against older persons. (pp. 163-199). Cambridge: Bradford Books.

McMullin, J. A., \& Marshall, V. W. (2001). Ageism, age relations, and garment industry work in Montreal. The Gerontologist, 41(1), 111-22. doi: $10.1093 /$ geront/41.1.111

Merriam-Webster (1994). The Merriam-Webster's Dictionary of English Usage. Massachusetts: G, \& C. Merriam Company.

Moura, R. M., \& Viana, H. B. (2011). Envelhecimento e preconceito: Uma análise da percepção de pessoas de meia-idade e idosos praticantes de atividades físicas. EFDeportes.com Revista Digital, 15(152). Recuperado de http://www.efdeportes.com/efd152/envelhecimento-epreconceito-percepcao-de-pessoas.htm

Nelson, T. D. (2005). Ageism: Prejudice against our feared future self. Journal of Social Issues, 61(2), 207-221. doi: 10.1111/j.15404560.2005.00402.x

Neri, A. L. (2007). Desenvolvimento e envelhecimento: perspectivas biológicas, psicológicas e sociológicas. $3^{\text {a }}$ edição. Campinas: Papirus.

Neri, A. L., \& Jorge, M. D. (2006). Atitudes e conhecimentos em relação à velhice em estudantes de graduação em educação e em saúde: subsídios ao planejamento curricular. Estudos de Psicologia, 23(2), 127-137. Recuperado de http://www.scielo.br/scielo.php?script=sci_ arttext\&pid $=$ S0103166X2006000200003\&lng $=$ pt\&nrm $=$ iso

Nussbaum, J. F., Pitts, M. J., Huber, F. N., Krieger, J. R. L., \& Ohs, J. E. (2005). Ageism and ageist language across the life span: Intimate relationships and non-intimate interactions. Journal of Social Issues, 61(2), 287-305. doi: 10.1111/j.1540-4560.2005.00406.x

Organização das Nações Unidas (1982). Assembléia Mundial sobre envelhecimento: Resolução 39/125. Viena: ONU.

Organização Mundial da Saúde (2002). The uses of epidemiology in the study of the elderly. Geneva: OMS. Recuperado de http://www.who.int/ iris/handle/10665/39136\#sthash.OsxRpCoM.dpuf

Palmore, E. B. (1999). Ageism: Negative and positive. New York: Springer Publishing Company.

Rodrigues, A., Assmar, E. M. L., \& Jablonski, B. (2000). Psicologia Social. $18^{a}$ edição. Rio de Janeiro: Vozes.

Torres, C. V., França, L. H. F., Oliveira, A., \& Presotti, L. (no prelo). Diagnóstico de diversidade cultural e inclusão nas organizações. Em H. Mendonça (Org.), Diagnóstico em organizações: Teoria e prática. São Paulo: Vetor.

Van Dalen, H., Henkens, K., \& Schippers, J. (2010). Productivity of older workers: Perceptions of employers and employees. Population and Development Review, 36(2), 309-330.

Wilkinson, J. A., \& Ferraro, K. F. (2002). Thirty years of ageism research. Em T. D. Nelson (Ed.), Ageism: Stereotyping and prejudice against older persons. (pp. 339-358). Cambridge: Bradford Books.

\section{Sobre os autores}

Andreia da Rocha Siqueira-Brito é Mestre em Psicologia pelo Programa de Pós-graduação da Universidade Salgado de Oliveira, bolsista da Coordenação de Aperfeiçoamento de Pessoal de Nível Superior.

Lucia Helena Freitas Pinho França é Professora Titular do Programa de Pós-graduação em Psicologia (Mestrado e Doutorado) da Universidade Salgado de Oliveira, com o apoio da Fundação de Amparo à Pesquisa do Estado do Rio de Janeiro (APQ1) e do Conselho Nacional de Desenvolvimento Científico e Tecnológico (bolsista produtividade 2).

Felipe Valentini é Professor Titular do Programa de Pós-graduação em Psicologia (Mestrado e Doutorado) da Universidade Salgado de Oliveira. 